\title{
Differential effects of the hypocretin 1 receptor antagonist SB 334867 on high-fat food self-administration and reinstatement of food seeking in rats
}

\author{
SG Nair, SA Golden and Y Shaham \\ Behavioral Neuroscience Branch, NIDA/IRP/NIH/DHHS, Baltimore, MD, USA
}

Background and purpose: Many studies have demonstrated a role of hypocretin 1 (orexin 1) receptors in home-cage food consumption in rodents. However, the role of these receptors in operant food self-administration or relapse to food seeking in animal models is unknown.

Experimental approach: In Experiment 1, we trained food-restricted rats (16-20 g per day) to lever press for high-fat (35\%) pellets (3-6 h per day, every other day). We then tested the effect of the hypocretin 1 receptor antagonist SB 334867 (10, $20 \mathrm{mg} \mathrm{kg}^{-1}$, i.p) on pellet self-administration. In Experiment 2, we trained rats to self-administer the food pellets, and following extinction of the food-reinforced responding, we tested the effect of hypocretin 1 ( 3 and $6 \mu$ g, i.c.v) on reinstatement of foodseeking and the effect of SB 334867 on this reinstatement. In Experiment 3, we tested the effect of SB 334867 on reinstatement induced by non-contingent pellet exposure (pellet-priming) or the pharmacological stressor yohimbine $\left(2 \mathrm{mg} \mathrm{kg}^{-1}\right.$, i.p).

Key results: SB 334867 attenuated high-fat pellet self-administration. In contrast, SB 334867 had no effect on reinstatement of lever presses induced by hypocretin 1, pellet-priming or yohimbine.

Conclusions and implications: These data indicate that during dieting, hypocretin 1 receptors contribute to operant high-fat pellet self-administration, but not to relapse to food seeking induced by acute re-exposure to the food itself or by the induction of a stress-like state.

British Journal of Pharmacology (2008) 154, 406-416; doi:10.1038/bjp.2008.3; published online 28 January 2008

Keywords: hypocretin; hypocretin 1 receptor; SB 334867; yohimbine; food priming; self-administration; reinstatement; relapse; orexin

Abbreviations: Hcrt 1, hypocretin type 1 receptor; Hcrt 2, hypocretin type 2 receptor; icv, intracerebroventricular i.p., intraperitoneal; SB 334867, 1-(2-methylbenzoxacol-6-yl)-3-[1,5]naphthyridin-4-yl urea hydrochloride

\section{Introduction}

High rates of relapse to maladaptive eating habits are a major problem in clinical treatment of obesity (Peterson and Mitchell, 1999). This relapse often occurs after re-exposure to palatable foods, food-associated cues or stress (Grilo et al., 1989; Drewnowski, 1997). The neuronal mechanisms underlying relapse during dietary treatments in humans are unknown. We recently adapted a reinstatement model, commonly used to study relapse to abused drugs (Stewart, 2000; Epstein et al., 2006), to examine the mechanisms underlying relapse to food seeking during dieting. In initial

Correspondence: Dr Y Shaham, Behavioral Neuroscience Branch, NIDA/IRP/ NIH, 5500 Nathan Shock Drive, Baltimore, MD 21224, USA.

E-mail: Yshaham@intra.nida.nih.gov

Received 31 October 2007; revised 29 November 2007; accepted 14 December 2007; published online 28 January 2008 studies, we found that systemic injections of a corticotropinreleasing factor 1 receptor antagonist blocks reinstatement of food seeking induced by the pharmacological stressor yohimbine, but has no effect on reinstatement induced by acute non-contingent exposure to food pellets (pelletpriming) (Ghitza et al., 2006). In contrast, systemic injections of peptide YY 3-36 blocked pellet-priming-induced reinstatement, but had no effect on yohimbine-induced reinstatement of food seeking (Ghitza et al., 2007). This pharmacological double dissociation suggests that different mechanisms mediate relapse induced by acute exposure to the previously self-administered food versus stress. Here, we further explored the mechanisms underlying food relapse by studying the role of hypocretin 1 (orexin 1) receptors in reinstatement of high-fat food seeking. 
Hypocretins (orexins) are neuropeptides synthesized by lateral hypothalamic and perifornical area neurons (de Lecea et al., 1998; Sakurai et al., 1998). The hypocretins comprise two distinct peptides, hypocretin 1 and 2 , and their effects are mediated by hypocretin type 1 (Hcrt 1) and type 2 (Hcrt 2) receptors (Sakurai et al., 1998). Hypocretin 1 has similar affinity for Hcrt 1 and Hcrt 2 receptors, whereas hypocretin 2 has a 10-fold greater affinity for Hcrt 2 than Hcrt 1 (Smart et al., 1999, 2000). These receptors are located on cell bodies of hypocretin neurons, and on pre- and postsynaptic terminals of these neurons (van den Pol et al., 1998). The hypocretin neurons project to neighbouring hypothalamic nuclei, as well as to various forebrain, midbrain and brainstem structures, consequently influencing several physiological processes (Peyron et al., 1998; Sutcliffe and de Lecea, 2002), including food intake (Sakurai et al., 1998). Intracerebroventricular (icv) injections of hypocretin 1 (and to a lesser extent hypocretin 2) increase home-cage food intake (Sakurai et al., 1998; Haynes et al., 1999; Rodgers et al., 2002). The stimulatory effect of hypocretin 1 on food intake is attenuated by the selective Hcrt 1 receptor antagonist 1-(2-methylbenzoxacol-6-yl)-3$[1,5]$ naphthyridin-4-yl urea hydrochloride (SB 334867) (Haynes et al., 2000; White et al., 2005). SB 334867 also decreases home-cage food consumption, indicating that activation of Hcrt 1 receptors regulates feeding (Haynes et al., 2002). More recently, Zheng et al. (2007) reported that ventricular and ventral tegmental area injections of SB 334867 attenuate the increase in high-fat food intake induced by $\mu$-opioid receptor activation in the nucleus accumbens.

Recent studies demonstrate that Hcrt 1 receptors play a role in drug reward, reinstatement of drug seeking and psychomotor sensitization (Harris and Aston-Jones, 2006). Concerning drug reward, Narita et al. (2006) reported that SB 334867 injections into the ventral tegmental area block the development of heroin conditioned place preference. Harris et al. (2007) demonstrated that unilateral lesion of lateral hypothalamic hypocretin neurons together with SB 334867 injections into the contralateral ventral tegmental area blocks the development of morphine conditioned place preference. Concerning reinstatement of drug seeking, Harris et al. (2005) reported that systemic injections of SB 334867 block reinstatement of morphine conditioned place preference induced by activation of lateral hypothalamic hypocretin neurons, Boutrel et al. (2005) found that SB 334867 attenuates footshock stress-induced reinstatement of cocaine seeking, and Lawrence et al. (2006) found that SB 334867 attenuates discriminative cue-induced reinstatement of alcohol seeking. Finally, Borgland et al. (2006) reported that systemic and ventral tegmental area SB 334867 injections attenuate cocaine psychomotor sensitization.

On the basis of these previous findings on the role of Hcrt 1 receptors on food intake and reinstatement of drug seeking, we assessed the effects of Hcrt 1 receptors on reinstatement of food seeking induced by hypocretin 1 , pellet-priming or yohimbine. Yohimbine is an $\alpha-2$ adrenoceptor antagonist that induces stress-like responses in human and laboratory animals (Bremner et al., 1996a,b). Yohimbine also reliably reinstates drug (Lee et al., 2004; Shepard et al., 2004; Le et al., 2005; Feltenstein and See, 2006; Marinelli et al., 2007) and food (Ghitza et al., 2006;
Nair et al., 2006) seeking in laboratory animals. Additionally, since prior studies only examined SB 334867's effects on home-cage food intake, we also assessed SB 334867's effect on operant high-fat (35\%) pellet self-administration.

\section{Materials and methods}

\section{Subjects and apparatus}

Male Long-Evans rats (total $n=80$; Charles River, Raleigh, NC, USA; 300-385 g) were housed in self-administration chambers for the duration of the experiment under a reverse $12 \mathrm{~h}: 12 \mathrm{~h}$ light-dark cycle (lights off at 0930 hours). A total of 10 rats were excluded from the study due to poor health $(n=6)$, failure to meet an extinction criterion $(n=3)$ and training failure $(n=1)$. The rats were kept on a restricted diet of $16 \mathrm{~g}$ per day of Purina rat chow (about $60-65 \%$ of their daily food intake) during the training phase, and on 16-20 g per day of this chow during the extinction and tests for reinstatement phases (see below). All procedures followed the guidelines outlined in the 'Principles of laboratory animal care' (NIH publication no. 85-23). Experiments were conducted in standard self-administration chambers (Med Associates, Georgia, VT, USA). Each chamber had two levers $9 \mathrm{~cm}$ above the floor, but only one lever ('active,' retractable lever) activated the pellet dispenser, which delivered 45-mg food pellets containing $35 \%$ fat and $45.2 \%$ carbohydrate (catalogue no. F05989; Bioserv, Frenchtown, NJ, USA).

\section{Drugs}

1-(2-Methylbenzoxacol-6-yl)-3-[1,5]naphthyridin-4-yl urea hydrochloride (Tocris Bioscience, Ellisville, MO, USA; or a generous donation from Eli Lilly) and yohimbine hydrochloride (Sigma, St Louis, MO, USA) were prepared fresh prior to testing. SB 334867 (10 and $20 \mathrm{mg} \mathrm{kg}^{-1}$, intraperitoneal (i.p.)) was dissolved in $10 \%$ 2-hydroxypropyl- $\beta$-cyclodextrin (encapsin) and 2\% dimethylsulphoxide (Sigma) and yohimbine $\left(2 \mathrm{mg} \mathrm{kg}^{-1}\right.$, i.p.) was dissolved in sterile distilled water. Hypocretin 1 (3 and $6 \mu \mathrm{g}$, icv, injection volume, $1-2 \mu \mathrm{l}$; Bachem, Torrence, CA, USA) was dissolved in $0.9 \%$ saline on the first day of testing. The peptide solution was stored at $4{ }^{\circ} \mathrm{C}$ for a maximum duration of 2 weeks. The injection volumes were $3 \mathrm{ml} \mathrm{kg}^{-1}$ i.p. for SB 334867 and $0.5 \mathrm{ml} \mathrm{kg}^{-1}$ i.p. for yohimbine. The doses for icv injections of hypocretin 1 and for systemic injections of yohimbine and SB 334867 were based on previous studies (Boutrel et al., 2005; Ghitza et al., 2006; Lawrence et al., 2006). The SB 334867 doses were also based on a pilot study $(n=7)$ where we tested the effect of vehicle or SB $334867\left(5,10,20\right.$ or $30 \mathrm{mg} \mathrm{kg}^{-1}$, i.p.) on the self-administration of chocolate-flavoured pellets. With the exception of the $5-\mathrm{mg} \mathrm{kg}^{-1}$ dose, all SB 334867 doses decreased pellet intake. However, injections of SB 334867 at a dose of $30 \mathrm{mg} \mathrm{kg}^{-1}$, but not lower doses, led to unwanted side effects (abnormal posture and immobility) that confound interpretation of lever pressing data using this dose.

\section{Intracranial surgery and intracranial injections}

The rats were anaesthetized with a mixture of sodium pentobarbital and chloral hydrate (60 and $25 \mathrm{mg} \mathrm{kg}^{-1}$, i.p.). 
They were implanted with guide cannulae (23-gauge; Plastics One, Roanoke, VA, USA) $2 \mathrm{~mm}$ above the right lateral ventricle (anterior-posterior: $-0.9 \mathrm{~mm}$, medial-lateral: $+1.4 \mathrm{~mm}$ and dorsal-ventral: $-2.0 \mathrm{~mm}$; Paxinos and Watson, 2005) using a stereotaxic instrument (Kopf, Tujunga, CA, USA). The analgesic buprenorphine ( $0.1 \mathrm{mg} \mathrm{kg}^{-1}$, subcutaneous) was given after surgery and the rats were allowed to recover for 7 days. Cannulae placements were verified by a positive dipsogenic response to angiotensin II (50 ng in $2 \mu \mathrm{l}$; Sigma). Placements were considered accurate if a rat started drinking within 2 min of the injection and sustained drinking for 3-4 min (Sakai et al., 1995). Icv injections of hypocretin 1 were made with Harvard infusion pumps, using 10- $\mu \mathrm{l}$ Hamilton syringes that were connected to 30-gauge injectors (Plastics One) via polyethylene-50 tubing. Injections lasted 1-2 min and injectors were left in place for an additional $1 \mathrm{~min}$ before being replaced with cannula blockers.

\section{Procedures}

With the exception of our initial experiment on the effect of SB 334867 on ongoing food pellet self-administration, we used a reinstatement procedure that included three phases as follows: training for food self-administration (9-14 sessions), extinction of the food-reinforced behaviour (10-16 sessions) and tests for reinstatement under extinction conditions (up to four sessions). Rats were chronically housed in the selfadministration chambers throughout the duration of the experiments. Separate groups of rats were used for each experiment. During all phases, the sessions started $30 \mathrm{~min}$ after the beginning of the dark cycle (1000 hours). In the following sections, we first describe the training and extinction procedures for all experiments, and then provide the specific details for the testing phase of each experiment. During testing, the experimental conditions were counterbalanced.

\section{Food self-administration training}

All rats were given 6-h daily sessions of 'autoshaping' for 2-3 days during which pellets were administered non-contingently every $5 \mathrm{~min}$ into a receptacle located near the active lever. Pellet delivery was accompanied by a compound 5-s tone $(2900 \mathrm{~Hz}, 15 \mathrm{~dB}$ above background)-light (a 7.5-W white light source located above the active lever) cue. Subsequently, the rats were trained to self-administer the pellets on a fixed-ratio-1, 20-s timeout reinforcement schedule. For experiment 1, the training sessions were conducted for 12-13 days, every other day, $6 \mathrm{~h}$ per day for 7 days (two 3-h sessions separated by $1 \mathrm{~h}$ ), followed by one 3-h per day session for 5-6 days (total: 24-26 days). For experiments 2 and 3 , the training sessions were conducted for 9-14 days, every other day (6-h per day in two 3-h sessions separated by $1 \mathrm{~h}$ ) (total: 18-28 days).

At the start of each 3-h session, the red houselight was turned on and the active lever was extended. Following each pellet delivery, the pellet cue was turned on for $5 \mathrm{~s}$. At the end of each 3-h session, the houselight was turned off and the active lever retracted. During the days on which the rats were not trained to lever press for food pellets, the $16 \mathrm{~g}$ regular food given at the start of the dark cycle.
We chose this training schedule and these diet conditions because previous home-cage food consumption studies have shown that rats placed on a restricted diet and given intermittent access to palatable food develop binge-like eating behaviour (Colantuoni et al., 2002; Corwin and Buda-Levin, 2004; Boggiano et al., 2007; Figlewicz et al., 2007; Avena et al., 2008), and become hypersensitive to the effect of stress on palatable food intake (Hagan et al., 2002, 2003). We also chose our diet conditions based on two other considerations. First, there is evidence that humans are particularly vulnerable to the effect of stress and food cues on relapse to maladaptive eating habits when they diet (Herman and Polivy, 1975; Fedoroff et al., 2003; Elfhag and Rossner, 2005). Second, as expected from these human studies, the effects of our experimental manipulations (in particular the pellet-priming condition) on reinstatement of food seeking are much less robust in rats trained, and tested while given free access to regular food (unpublished data).

\section{Extinction of food-reinforced responding}

For experiments 2 and 3, after training the rats were given 10-16 daily extinction sessions until active lever responding was below a mean of 30 presses per $3 \mathrm{~h}$ for three consecutive sessions (the extinction criterion). During the extinction phase, lever presses led to tone-light cue presentations, but not pellet delivery. Initially, the rats were given two 3 -h sessions (separated by $1 \mathrm{~h}$ ) each day for 6 days. Subsequently, they were given one 3 -h extinction session per day for additional 4-10 days until they met the extinction criterion of 30 or less active lever presses over three daily sessions. During the extinction and reinstatement phases, regular food (16-20 g) was given approximately at the same time as during training (that is, $\sim 7.5 \mathrm{~h}$ after the onset of the dark cycle). For rats with variable (unstable) extinction responding, the daily ration of Purina rat chow was increased to $20 \mathrm{~g}$ per day to ensure that they met the extinction criterion within 16 days; these rats were maintained on $20 \mathrm{~g}$ of Purina rat chow during the reinstatement tests.

Experiment 1: Effect of SB 334867 on pellet self-administration We studied the effect of systemic injections of SB 334867 on ongoing pellet self-administration. For 7 days, the rats $(n=16)$ were given two 3 -h self-administration training sessions as described above, following which they were given 5-6 days of one 3-h training session per day. We then assessed the effect of SB 334867 on pellet self-administration in three $3-\mathrm{h}$ tests that were conducted every $48-96 \mathrm{~h}$. We used a within-subject experimental design with the factors of SB 334867 dose (vehicle, 10 and $20 \mathrm{mg} \mathrm{kg}^{-1}$ ) and session hour (hours 1-3). The different drug doses were given in a counterbalanced order. SB 334867 or its vehicle was injected $60 \mathrm{~min}$ prior to the test sessions.

\section{Experiment 2: Effect of SB 334867 on hypocretin 1-induced reinstatement of food seeking}

The purpose of experiment 2 was to determine the effect of SB 334867 on hypocretin 1-induced reinstatement. We initially assessed the effect of hypocretin 1 on reinstatement of food 
seeking. Following self-administration training and extinction of lever presses, the rats $(n=9)$ were injected with vehicle or hypocretin 1 ( 3 and $6 \mu \mathrm{g}$ ) during three sessions, every other day, with extinction sessions on the intervening days. We used a within-subject experimental design with the factors of hypocretin 1 dose (vehicle, 3 and $6 \mu \mathrm{g}$ ) and session hour.

In a different group of rats $(n=28)$, we examined the effect of SB 334867 (10 and $20 \mathrm{mg} \mathrm{kg}^{-1}$, i.p., $n=10$ or 18 , respectively) on hypocretin 1 -induced reinstatement. We used a mixed experimental design that included the between-subject factor of SB 334867 dose (10 or $20 \mathrm{mg} \mathrm{kg}^{-1}$, $n=10$ or 18 , respectively) and the within-subject factors of pretreatment condition (0 and SB 334867 (10 or $\left.20 \mathrm{mg} \mathrm{kg}^{-1}\right)$ ), hypocretin 1 dose $(0$ and $6 \mu \mathrm{g}$ ) and session hour. On test days that were separated by $24-72 \mathrm{~h}$, each rat was injected systemically with the SB 334867 vehicle or one of the SB 334867 doses (10 or $20 \mathrm{mg} \mathrm{kg}^{-1}$ ) 45-60 min before the test sessions, and then injected with hypocretin 1 or its vehicle 30-35 min before the sessions; the injections of hypocretin 1 and its vehicle, and SB 334867 and its vehicle were counterbalanced.

Experiment 3: Effect of SB 334867 injections on pellet-priming- and yohimbine-induced reinstatement In experiment 3 we assessed the effect of SB 334867 on reinstatement induced by pellet-priming and yohimbine. These stimuli reliably reinstate lever presses under our experimental conditions (Ghitza et al., 2006, 2007; Nair et al., 2006).

Pellet-priming. We tested the effect of SB 334867 on pelletpriming-induced reinstatement in four 3-h test sessions, with two sessions run consecutively and one extinction day between sets of tests. During the test sessions, one food pellet was administered non-contingently just prior to the onset of the sessions and four other pellets were delivered 2 , 4, 6 and $8 \mathrm{~min}$ after the start of the sessions (total of five food pellets). We used a within-subject experimental design with the factors of SB 334867 dose (vehicle, $20 \mathrm{mg} \mathrm{kg}^{-1}$ ), pellet-priming (pellet, no pellet) and session hour $(n=8)$. Thus, each rat was given four counterbalanced test sessions: vehicle-no pellet, vehicle-pellet-priming, SB 334867-no pellet and SB 334867-pellet-priming. The rationale for using this experimental design is to minimize the number of rats used, while limiting the number of repeated pellet-priming reinstatement tests to minimize habituation to the pellet-priming effect over repeated testing. SB 334867 or its vehicle was injected $60 \mathrm{~min}$ before the start of the test sessions, and the experimental conditions were counterbalanced.

Yohimbine. We tested the effect of SB 334867 on yohimbineinduced reinstatement in four test sessions with two sessions run consecutively and one extinction day between sets of tests. We used a within-subject design with the factors of SB 334867 dose (vehicle, $20 \mathrm{mg} \mathrm{kg}^{-1}$ ), yohimbine dose (vehicle, $2 \mathrm{mg} \mathrm{kg}^{-1}$ ) and session hour $(n=9)$. The experimental conditions were counterbalanced. The rats were pretreated with SB 334867 or its vehicle, injected 60 min before the start of the test sessions and then injected with yohimbine or its vehicle $10-15 \mathrm{~min}$ after these injections.

\section{Statistical analyses}

Data were analysed using SPSS version 15.0; statistical software. The data on the effect of SB 334867 on pellet selfadministration (experiment 1) were analysed separately for the number of pellets self-administered and the number of timeout active lever presses per pellet; this ratio, derived by dividing the non-reinforced active lever presses by the number of pellets earned, provides an measure of the effect of SB 334867 on food-taking behaviour that is to some degree independent of the drug effect on pellet intake. The data from the reinstatement experiments (experiments 2 and 3) were analysed for non-reinforced lever presses on the previously active lever. The factors used in the statistical analyses are described in the Results section. The experimental manipulations had minimal effects on inactive lever presses (a mean of less than four presses per $3 \mathrm{~h}$ ), a potential measure of nondirected activity and/or response generalization (Shalev et al., 2002). Thus, the data for inactive lever presses are not reported in the Results section. Significant overall effects $(P<0.05)$ in the different analysis of variance tests were followed by post hoc Fisher's protected least-square detection tests.

\section{Results}

Experiment 1: Effect of SB 334867 injections on pellet self-administration

SB 334867 urea hydrochloride decreased the number of pellets self-administered (Figures 1a-c). In contrast, SB 334867 had no effect on the ratio of timeout active lever presses per self-administered pellet (Figure 1d). The rats $(n=16)$ were injected with vehicle or one dose of SB 334867 (10 or $20 \mathrm{mg} \mathrm{kg}^{-1}$ ) before three test sessions every $48-96 \mathrm{~h}$, during which the rats lever pressed for pellets. The statistical analyses for pellets self-administered included the within-subject factors of SB 334867 dose (vehicle, 10 and $20 \mathrm{mg} \mathrm{kg}^{-1}$ ) and session hour (hours 1-3). This analysis revealed significant effects of $\mathrm{SB} 334867$ dose $\left(\mathrm{F}_{2,30}=14.9\right.$, $P<0.01)$ and session hour $\left(\mathrm{F}_{2,30}=131.8, P<0.01\right)$; the latter effect is due to decreases in pellet intake during the session in all groups. The statistical analysis for the ratio of timeout active lever presses per self-administered pellet included the within-subject factor of SB 334867 dose. This analysis revealed no significant effect of SB 334867 dose $(P>0.3)$.

\section{Experiments 2 and 3: Effect of SB 334867 injections on} reinstatement

The rats in experiments 2 and 3 were trained for 9-14 sessions and demonstrated reliable pellet self-administration and, as in our previous studies, a progressive escalation of timeout active lever presses across sessions (Ghitza et al., 2006; Nair et al., 2006; Figure 2a). After food self-administration training, the rats were given six 6-h extinction sessions, and additional 3 -h extinction sessions during 

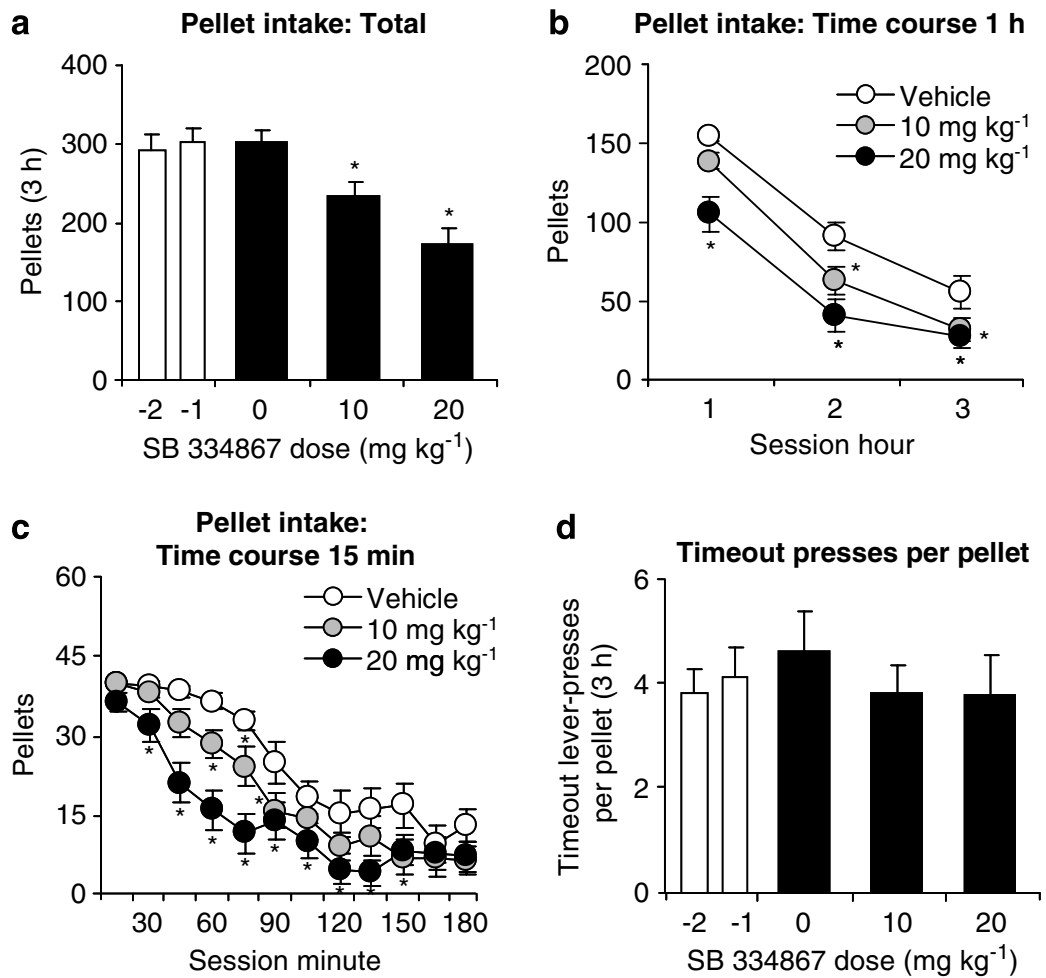

Figure 1 Systemic injections of SB 334867 decrease pellet self-administration. (a-c) Mean \pm s.e.mean number of $35 \%$ fat pellets selfadministered during two baseline days prior to testing $(-2$ and -1$)$ and after systemic injections of vehicle (10\% encapsin and $2 \%$ DMSO) or SB 334867 (total per $3 \mathrm{~h}$ and time course represented at 60- and 15-min intervals) $(n=16)$. (d) Mean ratio of timeout lever presses per selfadministered pellets during the two baseline days and after vehicle or SB 334867 injections. *Different from vehicle condition; $P<0.05$. DMSO, dimethylsulphoxide; SB 334867, 1-(2-methylbenzoxacol-6-yl)-3-[1,5]naphthyridin-4-yl urea hydrochloride.

which lever pressing decreased over time (Figure 2b). During the training phase, the statistical analyses revealed significant increases over time for both pellets earned and timeout active lever presses $(P$-values $<0.01)$, but not for inactive lever presses. During the extinction phase, the analyses revealed significant decreases over time for active lever presses during the first six extinction days during which the rats were given two 3-h daily sessions that were separated by $1 \mathrm{~h}(P$-values $<0.01)$. During self-administration training, the rats gained weight on the days in which the pellets were available and lost weight on the days in which the pellets were not available; during early extinction, the rats lost weight but subsequently maintained relatively stable body weight (Figure 2c). During the training phase, the rats gained approximately $35 \pm 3 \mathrm{~g}$ body weight, and their body weight on the first day of testing was $11 \pm 3 \mathrm{~g}$ greater than on the first training day.

\section{Hypocretin 1-induced reinstatement (experiment 2)}

Two groups of rats were tested. In the first group, we assessed the effect of ventricular injections of hypocretin 1 (3 and $6 \mu \mathrm{g}$ ) on reinstatement of food seeking. In the second group, we assessed the effect of SB 334867 (10 or $20 \mathrm{mg} \mathrm{kg}^{-1}$, i.p.) on reinstatement induced by hypocretin $1(6 \mu \mathrm{g})$. In the first group, the statistical analysis included the within-subject factor of hypocretin 1 dose (vehicle, 3 and $6 \mu \mathrm{g}$ ) and session hour. Hypocretin 1 increased lever pressing as indicated by significant effects of hypocretin 1 dose $\left(\mathrm{F}_{2,16}=5.4, P<0.05\right)$, and hypocretin 1 dose $\times$ session hour $\left(\mathrm{F}_{4,32}=5.8, P<0.01\right)$ (Figure 3a). In the second group, SB 334867 had no effect on hypocretin 1-induced reinstatement of lever responding (Figure 3c). The statistical analyses included the betweensubject factor of SB 334867 dose (10 or $20 \mathrm{mg} \mathrm{kg}^{-1}$ ) and within-subject factors of pretreatment condition (vehicle, SB 334867 (10 or $\left.20 \mathrm{mg} \mathrm{kg}^{-1}\right)$ ), hypocretin 1 dose $(0$ and $6 \mu \mathrm{g})$ and session hour. Analysis of variance revealed significant effects of hypocretin 1 dose $\left(\mathrm{F}_{1,27}=45.1, P<0.01\right)$ and hypocretin 1 dose $\times$ session hour $\left(F_{2,54}=31.9, P<0.01\right)$. There were no interactions between SB 334867 dose $\times$ hypocretin 1 dose or SB 334867 dose $\times$ hypocretin 1 dose $\times$ session hour $(P$-values $>0.1)$.

\section{Pellet-priming- and yohimbine-induced reinstatement (experiment 3)}

Systemic injections of SB 334867 had no effect on pelletpriming- or yohimbine-induced reinstatement of active lever responding (Figure 4). The statistical analyses included the within-subject factors of SB 334867 dose $\left(0\right.$ and $\left.20 \mathrm{mg} \mathrm{kg}^{-1}\right)$ and pellet-priming (pellet, no pellet) or yohimbine dose $(0$ and $2 \mathrm{mg} \mathrm{kg}^{-1}$ ). These analyses revealed significant effects of pellet-priming $\left(\mathrm{F}_{1,7}=7.1, P<0.05\right)$ and yohimbine dose $\left(\mathrm{F}_{1,8}=25.9, \quad P<0.01\right)$. The effect of session hour was significant for pellet-priming-induced reinstatement $(P<0.05)$, but not for yohimbine-induced reinstatement $(P>0.1)$. Neither the effect of SB 334867 dose nor the 

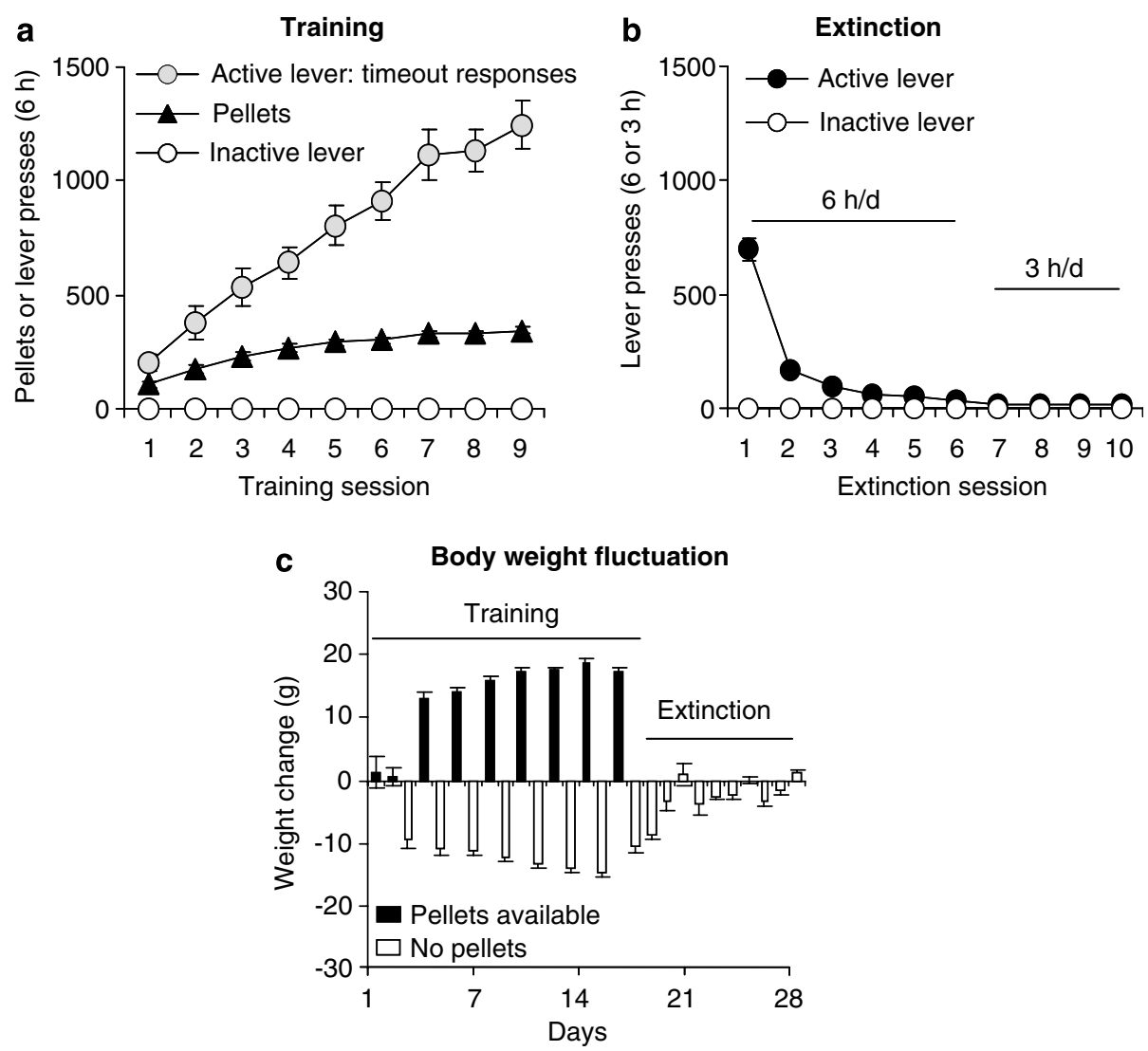

Figure 2 Food pellet self-administration training, extinction of the food-reinforced lever responding and body weight fluctuations. (a) Training: Mean \pm s.e.mean number of $35 \%$ fat pellets earned, timeout active lever presses and inactive lever presses during the training sessions over nine alternating days (two 3-h sessions/day, every other day) for rats that were trained under a fixed-ratio-1 (FR-1) 20-s timeout reinforcement schedule in experiments 2 and $3(n=54)$. (b) Extinction: Mean number of presses on the previously active lever or inactive lever during the extinction phase $(n=54)$. (c) Body weight: Mean daily weight fluctuation (in grams) during the training and extinction phases.

interaction between SB 334867 dose and pellet-priming or yohimbine dose was significant $(P$-values $>0.1)$.

\section{Discussion}

Two main findings emerge from the present experiments. First, systemic injections of the Hcrt 1 receptor antagonist SB 334867 significantly decreased (43\% at the high dose) operant high-fat pellet self-administration in food-restricted rats, indicating an important role of Hcrt 1 receptors in operant food self-administration. These findings extend previous reports on the effect of SB 334867 on home-cage food consumption (Rodgers et al., 2002). Second, systemic injections of SB 334867 had no effect on reinstatement of food seeking induced by hypocretin 1, pellet-priming and the pharmacological stressor yohimbine, suggesting that the Hcrt 1 receptor plays a minimal role in reinstatement of food seeking, as assessed in a rat relapse model (Stewart, 2000; Shaham et al., 2003; Epstein et al., 2006).

\section{Role of Hcrt 1 receptors in food self-administration}

We speculate that acceleration of satiety mechanisms underlie SB 334867's effects on high-fat pellet self-administration. Although SB 334867 decreased pellet intake at each hour of testing (Figure 1), it did not decrease pellet intake in the first $15 \mathrm{~min}$ of testing (Figure 1c). Thus, SB 334867 did not decrease the daily resumption of food-taking behaviour in hungry rats, but rather decreased food-taking behaviour after it was initiated, suggesting that the drug affected food satiety rather than the motivation to initiate food seeking. Additional support for the notion that SB 334867 accelerated food satiety rather than the motivation to seek food comes from the fact that the drug had no effect on pellet-priminginduced reinstatement of food seeking when the rats were only given a small amount of food (five pellets) at the onset of the sessions, but subsequent lever responding was not reinforced with food. Finally, the finding that SB 334867 had no effect on timeout non-reinforced lever presses, a potential index of the motivation to seek food in the presence of the tone-light pellet cue (Ghitza et al., 2006), is also in agreement with the satiety interpretation of SB 334867's effects.

A potential alternative interpretation of our data is that SB 334867 decreased food self-administration by inducing conditioned taste aversion. However, this possibility is unlikely because there is little evidence that the effect of SB 334867 on home-cage food consumption is due to taste aversion (Rodgers et al., 2002; Ishii et al., 2005b). In addition, hypocretins are critical modulators of the arousal state of the organism (Sutcliffe and de Lecea, 2002). Thus, another 

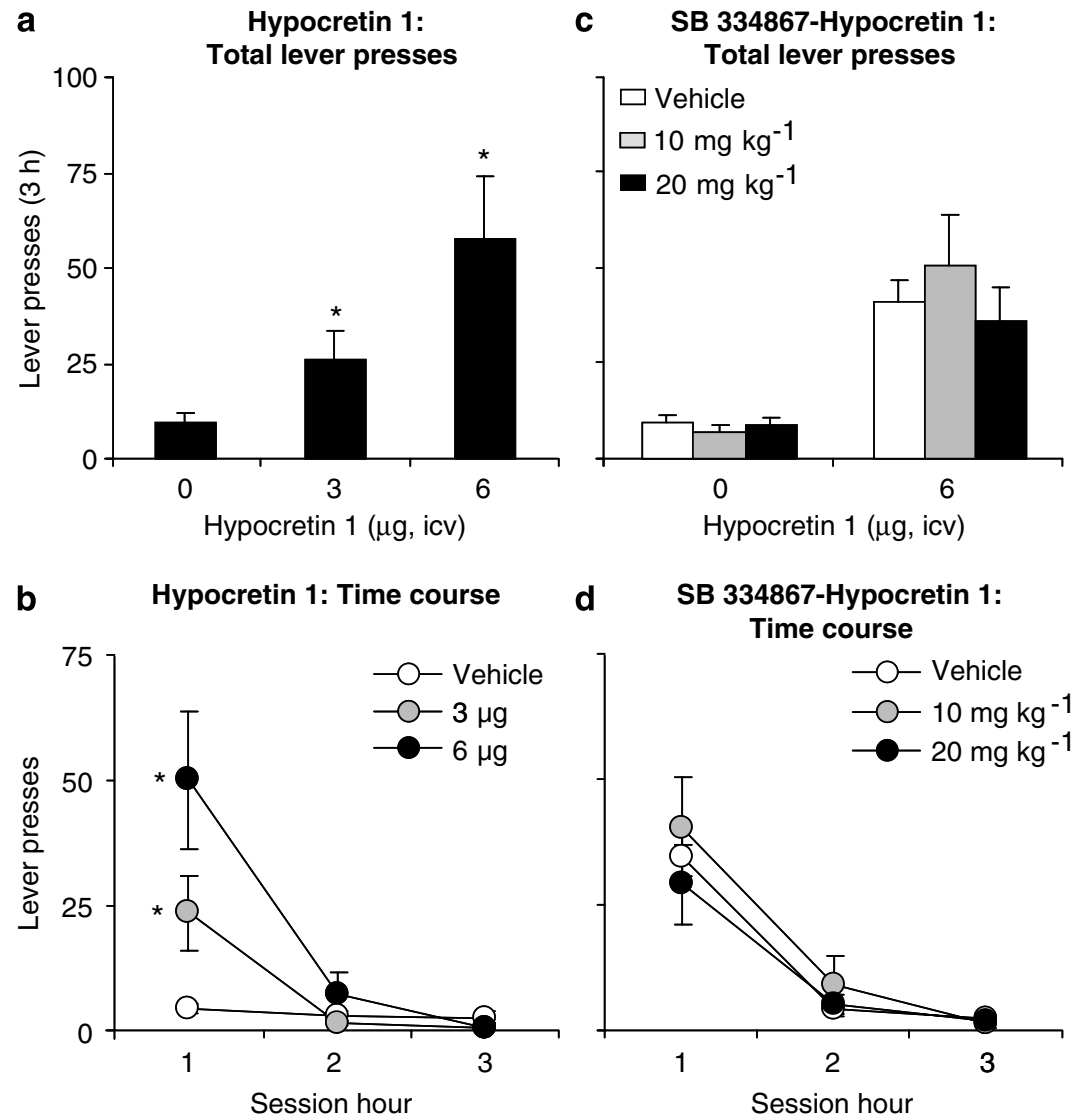

Figure 3 Systemic injections of SB 334867 had no effect on hypocretin 1-induced reinstatement of food seeking. (a) Mean \pm s.e.mean number of active lever presses after icv injections of vehicle (saline) and hypocretin 1 ( 3 and $6 \mu \mathrm{g}, n=9)$ during the reinstatement tests. *Different from vehicle condition; $P<0.05$. (b) Corresponding time course for the data described in (a). (c) Mean number of active lever presses in rats pretreated with SB 334867 ( $n=10-18$ per dose) or its vehicle $(n=28)$ prior to icv injections of hypocretin 1 or its vehicle. (d) Corresponding time course for the vehicle-hypocretin 1 and SB 334867-hypocretin 1 groups described in (c). *Different from vehiclehypocretin 1 condition; $P<0.05$. icv, intracerebroventricular; SB 334867, 1-(2-methylbenzoxacol-6-yl)-3-[1,5]naphthyridin-4-yl urea hydrochloride.

potential interpretation of our data is that the effect of SB 334867 on food self-administration is secondary to its effects on the rat's arousal state, resulting in non-selective disruption of motor performance. However, it is unlikely that SB 334867 interfered with motor performance in our study, because during training SB 334867 had no effect on timeout lever presses during the $20 \mathrm{~s}$ after pellet delivery, and during testing SB 334867 had no effect on reinstatement of food seeking induced by hypocretin 1, pellet-priming or yohimbine.

Thus, although multiple mechanisms may mediate the effect of pharmacological agents on food intake, the most likely explanation of the present results is that SB 334867 decreased operant food self-administration by accelerating satiety in hungry rats. This conclusion is congruent with the conclusion reached by Rodgers and co-workers in their studies on the effect of SB 334867 on home-cage food consumption (Rodgers et al., 2001, 2002; Ishii et al., 2005a).

Role of Hcrt 1 receptors in reinstatement of food seeking A question that is raised by the present results is what role hypocretin 1 plays in relapse to food seeking. On one hand, ventricular injections of hypocretin 1 reliably reinstated lever responding, suggesting a role of hypocretin 1 in relapse to food seeking. On the other hand, systemic injections of SB 334867, at doses that significantly decreased high-fat food self-administration, had no effect on reinstatement induced by pellet-priming, yohimbine or hypocretin 1 . These data potentially suggest that the role of hypocretin 1 in relapse to food seeking is mediated by Hcrt 2 receptors, an issue that can be explored pending the availability of selective Hcrt 2 receptor antagonists (Chang et al., 2007) or mixed Hcrt 1/ Hcrt 2 receptor antagonists (Brisbare-Roch et al., 2007). One possible explanation for the lack of effect of SB 334867 on reinstatement of food seeking is due to lower baseline response rates during tests for reinstatement versus selfadministration. However, this possibility is unlikely since the number of active lever presses in our reinstatement tests after exposure to hypocretin 1, pellet-priming and yohimbine are comparable to previous studies where SB 334867 decreased reinstatement of drug seeking (Boutrel et al., 2005; Lawrence et al., 2006). Alternatively, the negative findings with SB 334867 are due to methodological issues related to dose selection and the drug's bioavailability and duration of action. We believe that it is unlikely that these methodological issues compromise our data interpretation, because under our experimental conditions $20 \mathrm{mg} \mathrm{kg}^{-1}$ of SB 334867 

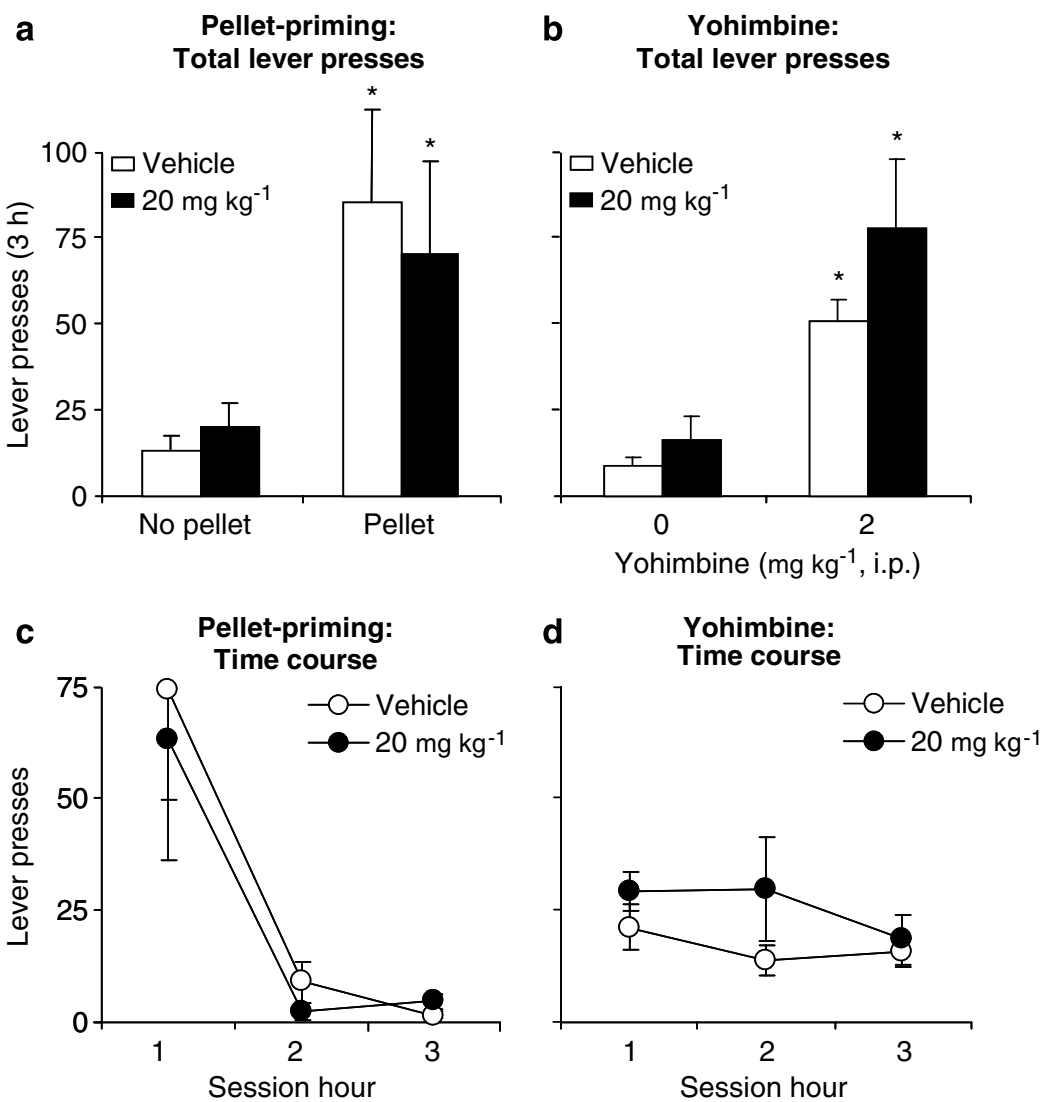

Figure 4 Systemic injections of SB 334867 had no effect on pellet-priming- or yohimbine-induced reinstatement of food seeking. (a) Pellet priming: Mean \pm s.e.mean number of active lever presses after pretreatment of SB 334867 or its vehicle and subsequent exposure to pelletpriming (delivery of 1 food pellet non-contingently every 2 min at the start of the session, total of five pellets) ( $n=8)$ or no pellet. (b) Yohimbine: Mean \pm s.e.mean number of active lever presses after pretreatment with SB 334867 or its vehicle and subsequent injections of yohimbine or its vehicle $(n=9)$. (c, d) Time course of pellet-priming- or yohimbine-induced reinstatement of lever responding. ${ }^{*}$ Different from the no pellet or the vehicle-yohimbine conditions within each SB 334867 condition (vehicle, $\left.20 \mathrm{mg} \mathrm{kg}^{-1}\right) ; P<0.05$. SB 334867, 1-(2methylbenzoxacol-6-yl)-3-[1,5]naphthyridin-4-yl urea hydrochloride.

decreased food self-administration in 15 of the 16 rats tested. However, because SB 334867 had no effect on reinstatement induced by three different stimuli, we discuss methodological issues related to the use of SB 334867 in detail in the following sections.

One issue to consider is that the SB 334867 doses used here (10 and $20 \mathrm{mg} \mathrm{kg}^{-1}$ ) were lower than the dose used in two previous studies in which SB $334867\left(30 \mathrm{mg} \mathrm{kg}^{-1}\right)$ was reported to decrease reinstatement of drug seeking induced by either intermittent footshock (Boutrel et al., 2005) or drug-priming injections (Harris et al., 2005). However, although our intention was to assess higher doses of SB 334867 in the reinstatement experiments, in our hands $30 \mathrm{mg} \mathrm{kg}^{-1}$ of the drug resulted in side effects that interfere with operant performance (see Materials and methods). Another issue to consider is that we injected SB 334867 45-60 min prior to the test sessions (previous studies mostly used 15-30 min injection-test intervals). In this regard, SB 334867 reaches maximal plasma and brain concentrations $30 \mathrm{~min}$ post injections, and has an elimination half-life of 2-4 $\mathrm{h}$ after i.p. injections (Ishii et al., 2005b). In addition, systemic injections of SB 334867 decrease both home-cage food consumption (Haynes et al., 2000; Ishii et al., 2005a; White et al., 2005) and body weight (Haynes et al., 2000) for at least $24 \mathrm{~h}$ after these injections. Together, it is unlikely that the lack of effect of SB 334867 on hypocretin 1-, pelletpriming or yohimbine-induced reinstatement of food seeking is due to our 45-60 min injection-test interval or the use of doses that are too low to have an impact on food-seeking behaviours.

Ventricular injections of hypocretin 1 robustly reinstated high-fat food seeking in food-restricted rats (Figure 3). This finding is somewhat different from that of Boutrel et al. (2005) who reported weak reinstatement of food seeking by hypocretin $1(6 \mu \mathrm{g}$, icv) in food-sated rats that were previously trained to lever press for $45 \mathrm{mg}$ low-fat chow pellets. One explanation for this discrepancy is the different type of food used (high- versus low-fat pellet). A more likely explanation for the more robust effect of hypocretin 1 on reinstatement in our experiment is the different feeding conditions (free-fed versus food restricted). Indeed, under our experimental conditions, yohimbine and pellet-priming are less effective stimuli for reinstatement of food seeking in sated rats (unpublished data).

A potential mechanism for the effect of hypocretin 1 on reinstatement is that it induces a more severe hunger state in our food-restricted rats, leading to increased motivation to seek food. In this regard, acute food deprivation $(24 \mathrm{~h})$ in 
food-sated rats reinstates heroin and cocaine seeking (Shalev et al., 2001, 2003, 2006), and also reinstates food seeking in food-sated rats (unpublished data). However, in foodrestricted rats under our training and testing conditions, omitting the daily $16-20 \mathrm{~g}$ ration had a minimal effect on reinstatement of lever presses (unpublished observations). This finding suggests that it is unlikely that exacerbation of hunger states is the mechanism underlying hypocretin 1-induced reinstatement.

In conclusion, we have not found evidence for a role of Hcrt 1 receptors in reinstatement of food seeking induced by pellet-priming, yohimbine and hypocretin 1 . The potent effect of hypocretin 1 on reinstatement, independent of Hcrt 1 receptor activation, suggests a potential role of Hcrt 2 receptors in this reinstatement. To the degree that reinstatement induced by hypocretin 2 involves in part neuronal systems that also mediate pellet-priming- or stress-induced reinstatement, activation of Hcrt 2 receptors may also contribute to reinstatement induced by these stimuli. Finally, on the basis of the findings of Lawrence et al. (2006) on the effect of SB 334867 on discriminative cueinduced reinstatement of alcohol seeking, a question for future research is whether this drug would also decrease cueinduced reinstatement of food seeking. The outcome of this study, however, is difficult to predict, because whereas discriminative cue- or context-induced reinstatement of drug seeking is associated with activation of lateral hypothalamus hypocretin neurons, context-induced reinstatement of food (sucrose) seeking is not (Hamlin et al., 2006, 2007; Dayas et al., 2008).

\section{Concluding remarks}

We found that the Hcrt 1 receptor antagonist SB 334867 decreased high-fat pellet self-administration, but had no effect on hypocretin 1-, pellet-priming- or yohimbineinduced reinstatement of food seeking. Previously, we found that the corticotropin-releasing factor 1 receptor antagonist antalarmin had no effect on pellet self-administration (unpublished data) or pellet-priming-induced reinstatement of food seeking, but decreased yohimbine-induced reinstatement (Ghitza et al., 2006). Recently, we found that peptide YY 3-36 had no effect on pellet self-administration or yohimbine-induced reinstatement of food seeking, but decreased pellet-priming-induced reinstatement (Ghitza et al., 2007). Thus, we have observed pharmacological double dissociations in the effects of different pharmacological agents on (1) high-fat operant self-administration (a measure of food reinforcement) versus reinstatement of food seeking during food restriction and (2) pellet-priming-induced reinstatement versus yohimbine-induced reinstatement. These pharmacological double dissociations suggest that (1) different neuronal mechanisms likely underlie food reinforcement and relapse to food seeking during dieting, and (2) different neuronal mechanisms underlie relapse to food seeking induced by acute re-exposure to the previously palatable food versus relapse induced by stress exposure. These conclusions are in agreement with previous findings that pharmacological agents that attenuate reinstatement of food seeking have minimal effects on ongoing food self-administration (de Vries et al., 2001; Baptista et al., 2004; De Vries et al., 2005; Bossert et al., 2006), and are consistent with results from studies in which drug self-administration and reinstatement models were used to assess mechanisms underlying drug reinforcement and relapse (De Vries and Shippenberg, 2002; Shalev et al., 2002; Kalivas and Volkow, 2005).

\section{Acknowledgements}

The work was supported by the Intramural Research Program of the National Institute on Drug Abuse. We thank Dr Elizabeth Duncan for helpful comments on the paper and Dr Luis deLecea for clarifying issues related to hypocretin structure, function and terminology. We also thank Sarah Gray for technical support and Eli Lilly for providing some of the SB 334867 used in this report.

\section{Conflict of interest}

The authors state no conflict of interest.

\section{References}

Avena NM, Rada P, Hoebel BG (2008). Evidence for sugar addiction: behavioral and neurochemical effects of intermittent, excessive sugar intake. Neurosci Biobehav Rev 32: 20-39.

Baptista MA, Martin-Fardon R, Weiss F (2004). Preferential effects of the metabotropic glutamate 2/3 receptor agonist LY379268 on conditioned reinstatement versus primary reinforcement: comparison between cocaine and a potent conventional reinforcer. J Neurosci 24: 4723-4727.

Boggiano MM, Artiga AI, Pritchett CE, Chandler-Laney PC, Smith ML, Eldridge AJ (2007). High intake of palatable food predicts binge-eating independent of susceptibility to obesity: an animal model of lean vs obese binge-eating and obesity with and without binge-eating. Int J Obes 31: 1357-1367.

Borgland SL, Taha SA, Sarti F, Fields HL, Bonci A (2006). Orexin A in the VTA is critical for the induction of synaptic plasticity and behavioral sensitization to cocaine. Neuron 49: 589-601.

Bossert JM, Poles GC, Sheffler-Collins SI, Ghitza UE (2006). The mGluR2/3 agonist LY379268 attenuates context- and discrete cueinduced reinstatement of sucrose seeking but not sucrose selfadministration in rats. Behav Brain Res 173: 148-152.

Boutrel B, Kenny PJ, Specio SE, Martin-Fardon R, Markou A, Koob GF et al. (2005). Role for hypocretin in mediating stress-induced reinstatement of cocaine-seeking behavior. Proc Natl Acad Sci USA 102: 19168-19173.

Bremner JD, Krystal JH, Southwick SM, Charney DS (1996a). Noradrenergic mechanisms in stress and anxiety: I. Preclinical studies. Synapse 23: 28-38.

Bremner JD, Krystal JH, Southwick SM, Charney DS (1996b). Noradrenergic mechanisms in stress and anxiety: II. Clinical studies. Synapse 23: 39-51.

Brisbare-Roch C, Dingemanse J, Koberstein R, Hoever P, Aissaoui H, Flores S et al. (2007). Promotion of sleep by targeting the orexin system in rats, dogs and humans. Nat Med 13: 150-155.

Chang H, Saito T, Ohiwa N, Tateoka M, Deocaris CC, Fujikawa T et al. (2007). Inhibitory effects of an orexin-2 receptor antagonist on orexin A- and stress-induced ACTH responses in conscious rats. Neurosci Res 57: 462-466.

Colantuoni C, Rada P, McCarthy J, Patten C, Avena NM, Chadeayne A et al. (2002). Evidence that intermittent, excessive sugar intake causes endogenous opioid dependence. Obes Res 10: 478-488.

Corwin RL, Buda-Levin A (2004). Behavioral models of binge-type eating. Physiol Behav 82: 123-130. 
Dayas CV, McGranahan TM, Martin-Fardon R, Weiss F (2008). Stimuli linked to ethanol availability activate hypothalamic CART and orexin neurons in a reinstatement model of relapse. Biol Psychiatry 63: 152-157.

de Lecea L, Kilduff TS, Peyron C, Gao X, Foye PE, Danielson PE et al. (1998). The hypocretins: hypothalamus-specific peptides with neuroexcitatory activity. Proc Natl Acad Sci USA 95: 322-327.

De Vries TJ, de Vries W, Janssen MC, Schoffelmeer AN (2005). Suppression of conditioned nicotine and sucrose seeking by the cannabinoid-1 receptor antagonist SR141716A. Behav Brain Res 161: 164-168.

de Vries TJ, Shaham Y, Homberg JR, Crombag H, Schuurman K, Dieben J et al. (2001). A cannabinoid mechanism in relapse to cocaine seeking. Nat Med 7: 1151-1154.

De Vries TJ, Shippenberg TS (2002). Neural systems underlying opiate addiction. J Neurosci 22: 3321-3325.

Drewnowski A (1997). Taste preferences and food intake. Annu Rev Nutr 17: 237-253.

Elfhag K, Rossner S (2005). Who succeeds in maintaining weight loss? A conceptual review of factors associated with weight loss maintenance and weight regain. Obes Rev 6: 67-85.

Epstein DH, Preston KL, Stewart J, Shaham Y (2006). Toward a model of drug relapse: an assessment of the validity of the reinstatement procedure. Psychopharmacology 189: 1-16.

Fedoroff I, Polivy J, Herman CP (2003). The specificity of restrained versus unrestrained eaters' responses to food cues: general desire to eat, or craving for the cued food? Appetite 41: 7-13.

Feltenstein MW, See RE (2006). Potentiation of cue-induced reinstatement of cocaine-seeking in rats by the anxiogenic drug yohimbine. Behav Brain Res 174: 1-8.

Figlewicz DP, MacDonald Naleid A, Sipols AJ (2007). Modulation of food reward by adiposity signals. Physiol Behav 91: 473-478.

Ghitza UE, Gray SM, Epstein DH, Rice KC, Shaham Y (2006). The anxiogenic drug yohimbine reinstates palatable food seeking in a rat relapse model: a role of $\mathrm{CRF}(1)$ receptors. Neuropsychopharmacology 31: 2188-2196.

Ghitza UE, Nair SG, Golden SA, Gray SM, Uejima JL, Bossert JM et al. (2007). Peptide YY3-36 decreases reinstatement of high-fat food seeking during dieting in a rat relapse model. J Neurosci 27: 11522-11532.

Grilo CM, Shiffman S, Wing RR (1989). Relapse crises and coping among dieters. J Consult Clin Psychol 57: 488-495.

Hagan MM, Chandler PC, Wauford PK, Rybak RJ, Oswald KD (2003). The role of palatable food and hunger as trigger factors in an animal model of stress induced binge eating. Int J Eat Disord 34: 183-197.

Hagan MM, Wauford PK, Chandler PC, Jarrett LA, Rybak RJ, Blackburn K (2002). A new animal model of binge eating: key synergistic role of past caloric restriction and stress. Physiol Behav 77: $45-54$.

Hamlin AS, Blatchford KE, McNally GP (2006). Renewal of an extinguished instrumental response: neural correlates and the role of D1 dopamine receptors. Neuroscience 143: 25-38.

Hamlin AS, Newby J, McNally GP (2007). The neural correlates and role of D1 dopamine receptors in renewal of extinguished alcoholseeking. Neuroscience 146: 525-536.

Harris GC, Aston-Jones G (2006). Arousal and reward: a dichotomy in hypocretin function. Trends Neurosci 29: 571-577.

Harris GC, Wimmer M, Aston-Jones G (2005). A role for lateral hypothalamic orexin neurons in reward seeking. Nature 437: 556-559.

Harris GC, Wimmer M, Randall-Thompson JF, Aston-Jones G (2007). Lateral hypothalamic orexin neurons are critically involved in learning to associate an environment with morphine reward. Behav Brain Res 183: 43-51.

Haynes AC, Chapman H, Taylor C, Moore GB, Cawthorne MA, Tadayyon $\mathrm{M}$ et al. (2002). Anorectic, thermogenic and anti-obesity activity of a selective orexin-1 receptor antagonist in ob/ob mice. Regul Pept 104: 153-159.

Haynes AC, Jackson B, Chapman H, Tadayyon M, Johns A, Porter RA et al. (2000). A selective orexin-1 receptor antagonist reduces food consumption in male and female rats. Regul Pept 96: 45-51.

Haynes AC, Jackson B, Overend P, Buckingham RE, Wilson S, Tadayyon $\mathrm{M}$ et al. (1999). Effects of single and chronic intracerebroventricular administration of the orexins on feeding in the rat. Peptides 20: 1099-1105.

Herman CP, Polivy J (1975). Anxiety, restraint, and eating behavior. J Abnorm Psychol 84: 66-72.

Ishii Y, Blundell JE, Halford JC, Upton N, Porter R, Johns A et al. (2005a). Anorexia and weight loss in male rats $24 \mathrm{~h}$ following single dose treatment with orexin-1 receptor antagonist SB-334867. Behav Brain Res 157: 331-341.

Ishii Y, Blundell JE, Halford JC, Upton N, Porter R, Johns A et al. (2005b). Satiety enhancement by selective orexin-1 receptor antagonist SB-334867: influence of test context and profile comparison with CCK-8S. Behav Brain Res 160: 11-24.

Kalivas PW, Volkow ND (2005). The neural basis of addiction: a pathology of motivation and choice. Am J Psychiatry 162: 1403-1413.

Lawrence AJ, Cowen MS, Yang HJ, Chen F, Oldfield B (2006). The orexin system regulates alcohol-seeking in rats. Br J Pharmacol 148: 752-759.

Le AD, Harding S, Juzytsch W, Funk D, Shaham Y (2005). Role of alpha-2 adrenoceptors in stress-induced reinstatement of alcohol seeking and alcohol self-administration in rats. Psychopharmacology 179: 366-373.

Lee B, Tiefenbacher S, Platt DM, Spealman RD (2004). Pharmacological blockade of alpha(2)-adrenoceptors induces reinstatement of cocaine-seeking behavior in squirrel monkeys. Neuropsychopharmacology 29: 686-693.

Marinelli PW, Funk D, Juzytsch W, Harding S, Rice KC, Shaham Y et al. (2007). The CRF(1) receptor antagonist antalarmin attenuates yohimbine-induced increases in operant alcohol self-administration and reinstatement of alcohol seeking in rats. Psychopharmacology 195: 345-355.

Nair SG, Gray SM, Ghitza UE (2006). Role of food type in yohimbineand pellet-priming-induced reinstatement of food seeking. Physiol Behav 88: 559-566.

Narita M, Nagumo Y, Hashimoto S, Narita M, Khotib J, Miyatake M et al. (2006). Direct involvement of orexinergic systems in the activation of the mesolimbic dopamine pathway and related behaviors induced by morphine. J Neurosci 26: 398-405.

Paxinos G, Watson C (2005). The Rat Brain in Stereotaxic Coordinates. Elsevier Academic Press: Amsterdam.

Peterson CB, Mitchell JE (1999). Psychosocial and pharmacological treatment of eating disorders: a review of research findings. J Clin Psychol 55: 685-697.

Peyron C, Tighe DK, van den Pol AN, de Lecea L, Heller HC, Sutcliffe JG et al. (1998). Neurons containing hypocretin (orexin) project to multiple neuronal systems. J Neurosci 18: 9996-10015.

Rodgers RJ, Halford JC, Nunes de Souza RL, Canto de Souza AL, Piper DC, Arch JR et al. (2001). SB-334867, a selective orexin-1 receptor antagonist, enhances behavioural satiety and blocks the hyperphagic effect of hypocretin-A in rats. Eur J Neurosci 13: $1444-1452$.

Rodgers RJ, Ishii Y, Halford JC, Blundell JE (2002). Orexins and appetite regulation. Neuropeptides 36: 303-325.

Sakai RR, Ma LY, He PF, Fluharty SJ (1995). Intracerebroventricular administration of angiotensin type 1 (AT1) receptor antisense oligonucleotides attenuate thirst in the rat. Regul Pept 59: 183-192.

Sakurai T, Amemiya A, Ishii M, Matsuzaki I, Chemelli RM, Tanaka $\mathrm{H}$ et al. (1998). Orexins and orexin receptors: a family of hypothalamic neuropeptides and $G$ protein-coupled receptors that regulate feeding behavior. Cell 92: 573-585.

Shaham Y, Shalev U, Lu L, De Wit H, Stewart J (2003). The reinstatement model of drug relapse: history, methodology and major findings. Psychopharmacology 168: 3-20.

Shalev U, Finnie PS, Quinn T, Tobin S, Wahi P (2006). A role for corticotropin-releasing factor, but not corticosterone, in acute food-deprivation-induced reinstatement of heroin seeking in rats. Psychopharmacology 187: 376-384.

Shalev U, Grimm JW, Shaham Y (2002). Neurobiology of relapse to heroin and cocaine seeking: a review. Pharmacol Rev 54: 1-42.

Shalev U, Marinelli M, Baumann MH, Piazza PV, Shaham Y (2003). The role of corticosterone in food deprivation-induced reinstatement of cocaine seeking in the rat. Psychopharmacology 168: 170-176. 
Shalev U, Yap J, Shaham Y (2001). Leptin attenuates food deprivation-induced relapse to heroin seeking. J Neurosci 21: RC129.

Shepard JD, Bossert JM, Liu SY, Shaham Y (2004). The anxiogenic drug yohimbine reinstates methamphetamine seeking in a rat model of drug relapse. Biol Psychiatry 55: 1082-1089.

Smart D, Jerman JC, Brough SJ, Neville WA, Jewitt F, Porter RA (2000). The orexins are weak agonists at recombinant human orexin-1 and orexin-2 receptors. Br J Pharmacol 129: 1289-1291.

Smart D, Jerman JC, Brough SJ, Rushton SL, Murdock PR, Jewitt F et al. (1999). Characterization of recombinant human orexin receptor pharmacology in a Chinese hamster ovary cell-line using FLIPR. Br J Pharmacol 128: 1-3.

Stewart J (2000). Pathways to relapse: the neurobiology of drug- and stress-induced relapse to drug-taking. J Psychiatry Neurosci 25: 125-136.
Sutcliffe JG, de Lecea L (2002). The hypocretins: setting the arousal threshold. Nat Rev Neurosci 3: 339-349.

van den Pol AN, Gao XB, Obrietan K, Kilduff TS, Belousov AB (1998). Presynaptic and postsynaptic actions and modulation of neuroendocrine neurons by a new hypothalamic peptide, hypocretin/ orexin. J Neurosci 18: 7962-7971.

White CL, Ishii Y, Mendoza T, Upton N, Stasi LP, Bray GA et al. (2005). Effect of a selective OX1R antagonist on food intake and body weight in two strains of rats that differ in susceptibility to dietary-induced obesity. Peptides 26: 2331-2338.

Zheng H, Patterson LM, Berthoud HR (2007). Orexin signaling in the ventral tegmental area is required for high-fat appetite induced by opioid stimulation of the nucleus accumbens. I Neurosci 27: $11075-11082$. 